\title{
Emergence of long-range magnetic order stabilized by magnetic impurities in pnictides
}

\author{
Carla Lupo, Thomas Julian Roberts, and Cedric Weber \\ Theory and Simulation of Condensed Matter, The Strand, King's College London, WC2R 2LS London, United Kingdom
}

(Received 15 November 2018; revised manuscript received 20 March 2019; published 26 June 2019)

\begin{abstract}
The Mermin-Wagner theorem prevents the stabilization of long-range magnetic order in two-dimensional layered materials, such as the pnictide superconductors, unless the magnetism is associated with a discrete symmetry breaking. A typical known example is the discrete row and column collinear magnetic state that emerges in doped iron pnictide materials due to an order-by-disorder mechanism. In these compounds, the magnetic state competes with superconductivity and the mechanism that stabilizes magnetism remains controversial. In this work, we report the phase diagram of a doped frustrated Heisenberg model obtained through Monte Carlo simulations combined with a parallel tempering simulation technique. The emergence of long-range magnetic order is stabilized by interactions between the magnetic dopant impurities.
\end{abstract}

DOI: 10.1103/PhysRevB.99.214439

\section{INTRODUCTION}

Since unconventional superconductivity occurs in the proximity of magnetically ordered states in many materials [1,2], understanding the magnetic phase of the parent compound is an important step toward understanding the mechanism of superconductivity. While for cuprates magnetism the underlying electronic state is understood, there is still debate in the case of the pairing mechanism in iron pnictides $\mathrm{BaFe}_{2} \mathrm{As}_{2}$ [3]. Many low-energy probes such as resistivity [4], scanning tunneling microscopy [5], and angle-resolved photoemission spectroscopy [6] have measured strong in-plane anisotropy of the electronic states, but there is no consensus on its physical origin. It was suggested from first-principles calculations [7] that the origin stems from orbital order, but the obtained anisotropy in the resistivity is opposite to the one found experimentally [8]. A more likely scenario supported by recent neutron diffraction measurements [9] is related to a spin density wave instability due to the presence of electron and hole pockets around $\mathbf{k}=(\pi, 0)$ and $\mathbf{k}=(0, \pi)$. The resulting magnetic order is of nematic type and can be seen as a helicoidal magnetic state with pitch vector $\mathbf{Q}=(0, \pi)$ or $\mathbf{Q}=(\pi, 0)$.

It has been suggested both experimentally $[10,11]$ and theoretically [12] that impurities have a dramatic impact on the magnetic and superconducting properties. Recent observation of the collinear magnetic phase has been reported in $\mathrm{Mn}$ doped La1111 iron-based superconductors [13] induced by the $\mathrm{Mn}$ impurities. The magnetic state induced by $\mathrm{Mn}$ and $\mathrm{Fe}$ substitutions in F-doped $\mathrm{LaFe}_{1-x} \mathrm{Mn}_{x} \mathrm{AsO}$ superconductors, reveals a fast drop of superconductivity and the recovery of a magnetic ground state at low doping, which have been

Published by the American Physical Society under the terms of the Creative Commons Attribution 4.0 International license. Further distribution of this work must maintain attribution to the author(s) and the published article's title, journal citation, and DOI. attributed to Ruderman-Kittel-Kasuya-Yosida interactions [14]. Furthermore, a new type of magnetic order due to the presence of magnetic impurities in $\mathrm{BaFe}_{2} \mathrm{As}_{2}$ [15] emerges in magnetic polarized x-ray measurements. Additionally, in recent studies of optimally electron-doped $\mathrm{CaKFe}_{4} \mathrm{As}_{4}$ [16] a novel magnetic order state, called spin-vortex crystal [17,18], different from the stripe antiferromagnetic or nematic phase, has been observed as the result of the magnetic fluctuations near the $(\pi, \pi) \mathbf{Q}$ vectors.

In this work we clarify the interaction of frustrated magnetic systems with impurities and in particular the double-Q state of the canonical $J_{1}-J_{2}$ model.

To describe the low-energy magnetic properties of this system, it has been suggested early on that a local moment picture may become relevant in the presence of moderately large electronic correlations [19], leading to the Heisenberg model with both nearest $\left(J_{1}\right)$ and next-nearest $\left(J_{2}\right)$ exchange couplings defined by

$$
\hat{\mathcal{H}}=J_{1} \sum_{\langle i, j\rangle} \hat{\mathbf{S}}_{i} \cdot \hat{\mathbf{S}}+J_{2} \sum_{\langle\langle i, j\rangle\rangle} \hat{\mathbf{S}}_{i} \cdot \hat{\mathbf{S}}_{j} .
$$

In the collinear regime, both $J_{1}$ and $J_{2}$ are positive, and $2 J_{2}>J_{1}$ [20]. In this expression, $\hat{\mathbf{S}}_{i}$ are $\mathrm{O}(3)$ spins on a periodic square lattice with $N=L \times L$ sites. $\langle i, j\rangle$ and $\langle\langle i, j\rangle\rangle$ indicate the sum over nearest and next-nearest neighbors, respectively. ${ }^{1}$

The first attempt at fitting the experimental spin density wave excitation spectra with a Heisenberg model suggested that one should use very anisotropic values of $J_{1}$ [21] and therefore it was argued [22] that to get a proper description of magnetic interactions and spin fluctuations in ferropnictides, additional biquadratic interactions might be important. However, it was later shown that the fits of the experimental data included energy scales beyond $100 \mathrm{meV}$, which are not well

\footnotetext{
${ }^{1} J_{1}$ sets the energy scale, and in our work we use $J_{2} / J_{1}=0.55$ when not specified otherwise, and both $J_{1}>0$ and $J_{2}>0$.
} 
described by magnon excitations [23]. A more careful study, including the itinerant character of the electrons [24], led to the conclusion that pnictides are indeed in the collinear regime with $[\mathbf{Q}=(0, \pi),(\pi, 0)]$ magnetic instabilities, a conclusion supported by first-principles calculations for selenium-based compounds $\left(\mathrm{KFe}_{2} \mathrm{Se}_{2}\right)$ [25]. All these results call for an indepth investigation of the effect of impurities in this frustrated Heisenberg model.

In this work we build upon our earlier results in Ref. [26] by extending the calculations to samples doped with both magnetic and nonmagnetic impurities, exploring highly doped lattices (up to full doping). In particular, we focus on the competing magnetic order at high doping, which corresponds to optimally and overdoped pnictide samples. We address the question of the interplay between the frustration induced by the exchange couplings and the disorder induced by the imperfections of the crystallographic structure. Increasing the doping we expect the possibility of first-order phase transitions driven by a percolation mechanism, where impurities drive local fluctuating order parameters on short distances and become long range at high dilutions.

\section{METHOD}

Since density functional calculations, and quite generally quantum-based calculations, are limited to relatively small unit cells and cannot tackle the issue of large supercell structures, we limit our calculations to a frustrated classical model [27], and carry out Monte Carlo calculations of the Heisenberg $J_{1}-J_{2}$ model in the presence of impurities extending the numerical approach in Refs. [28,29] with the implementation of the parallel tempering simulation methods. Indeed this replica exchange method has the key role in sampling the phase space at low temperature preventing the systems from being trapped in local minima. Further details are reported in the Supplemental Material [30] (see Sec. B, and references therein [31-34]).

In our calculations we consider samples doped with both magnetic and nonmagnetic impurities, exploring highly doped lattices. The doping is implemented in our algorithm replacing a fraction of lattice sites with impurities. The concentration of the doping is indicated by $\delta=N_{\text {imp }} / N_{\text {tot }}$ with $N_{\text {imp }}$ being the number of impurities and $N_{\text {tot }}=L \times L$ is the total number of sites in the lattice. For a fixed doping value, the corresponding fraction of sites in the lattice $S_{\text {lat }}$ are randomly selected and replaced with impurities $S_{\text {imp. }}$. Hence we are considering a site dilution disorder type. An example of a different type of disorder (bond dilution) is provided by Ref. [35]. We note, however, that the consequent magnetic phases detected are different respectively to the type of disordered imposed.

The magnetic moment of the impurities $S_{\mathrm{imp}}$ is characterized by its ratio with the magnetic moment of the undoped compound (e.g., $S_{\mathrm{Fe}}$ for iron), $r=S_{\mathrm{imp}} / S_{\mathrm{Fe}}$.

The impurity spins are not quenched but instead are energetically optimized by the heatbath algorithm [36,37]. The physical observables computed are averaged over a large number of disordered configurations (up to 5000 configurations) by using a BlueGene/Q supercomputer facility.
We limit ourselves to $L \times L=50 \times 50$ cluster size and we impose periodic boundary conditions. Our system size selection follows from preliminary investigation of the finite size effect on the order parameters reported in the Supplemental Material Fig. S3.

\section{MAGNETIC ORDER IN THE UNDOPED SYSTEM}

In the absence of disorder and at zero temperature, the magnetic vector is $\mathbf{Q}=(\pi, \pi)$ for $J_{2} / J_{1}<0.5$, and for $J_{2} / J_{1}>0.5$ the ground state is continuously degenerate and is characterized by a bipartite lattice, with two distinct antiferromagnetically ordered states on each sublattice, with $\theta$ the angle between the two magnetic directions. At finite temperature the entropy selection reduces the $\mathrm{O}(3)$ symmetry of the ground state to $Z_{2}$ selecting the states with antiferromagnetic spin correlations in one spatial direction and ferromagnetic correlations in the other $[\mathbf{Q}=(0, \pi),(\pi, 0)]$. This is the socalled order-by-disorder entropic selection and the associated discrete symmetry breaking drives a finite temperature Isinglike phase transition $[26,38]$. We address how the presence of disorder affects this transition.

\section{INVESTIGATION OF DOPED SYSTEM}

In the following discussion we report the phase diagram of a doped frustrated Heisenberg model obtained through Monte Carlo simulations combined with a parallel tempering (replica exchange) simulation technique. We introduce both magnetic $(r \neq 0)$ and nonmagnetic disorder $(r=0)$ and we compare the behavior of the emerging magnetic phases focusing on whether a long-range magnetic order is stabilized by interactions between the magnetic dopant impurities.

Effect of doping on the collinear order. In Fig. 1 we consider the collinear order parameter constructed from the original spin variables $\hat{\mathbf{S}}_{i}$,

$$
M_{2}(x)=\left(\hat{\mathbf{S}}_{i}-\hat{\mathbf{S}}_{k}\right) \cdot\left(\hat{\mathbf{S}}_{j}-\hat{\mathbf{S}}_{l}\right),
$$

where $(i, j, k, l)$ are the corners with diagonal $(i, k)$ and $(j, l)$ of the plaquette centered at the site $x$ of the dual lattice [see Supplemental Material Fig. S1(a)], and we define its normalized counterpart as $Z_{2}(x)=M_{2}(x) /\left|M_{2}(x)\right|$. In this way, the two collinear states with $\mathbf{Q}=(\pi, 0)$ and $\mathbf{Q}=(0, \pi)$ can be distinguished by the value of the Ising variable, $Z_{2}(x)= \pm 1$.

For impurities with a 50\% larger magnetic moment [see Fig. 1(b)], we observe that there exists a temperature range $T=(0.1,0.2) J_{1}$ where the collinear order survives at all dilutions. However, the transition from collinear to paramagnetic (from region $\mathrm{B}$ to $\mathrm{C}$ ) at high temperature increases from 0.2 to 0.45 . This can be explained by a very simple argument; in the fully doped regime all spins are 1.5 times larger and so the energy scales are rescaled by a factor $1.5^{2}$, increasing $T_{c}$ in turn by a factor 2.25. A different behavior of the collinear order is observed considering the same temperature range, $T=(0.1,0.2) J_{1}$, either if we increase $(r=2)$ or decrease $(r=0)$ the magnetic moment of the dopant. Indeed the Ising-like order is rapidly suppressed by doping with nonmagnetic $r=0$ impurities [Fig. 1(a)] or impurities with a large magnetic moment $r=2$ [Fig. 1(c)], with no collinear magnetic order obtained beyond $8 \%$ dilution. 

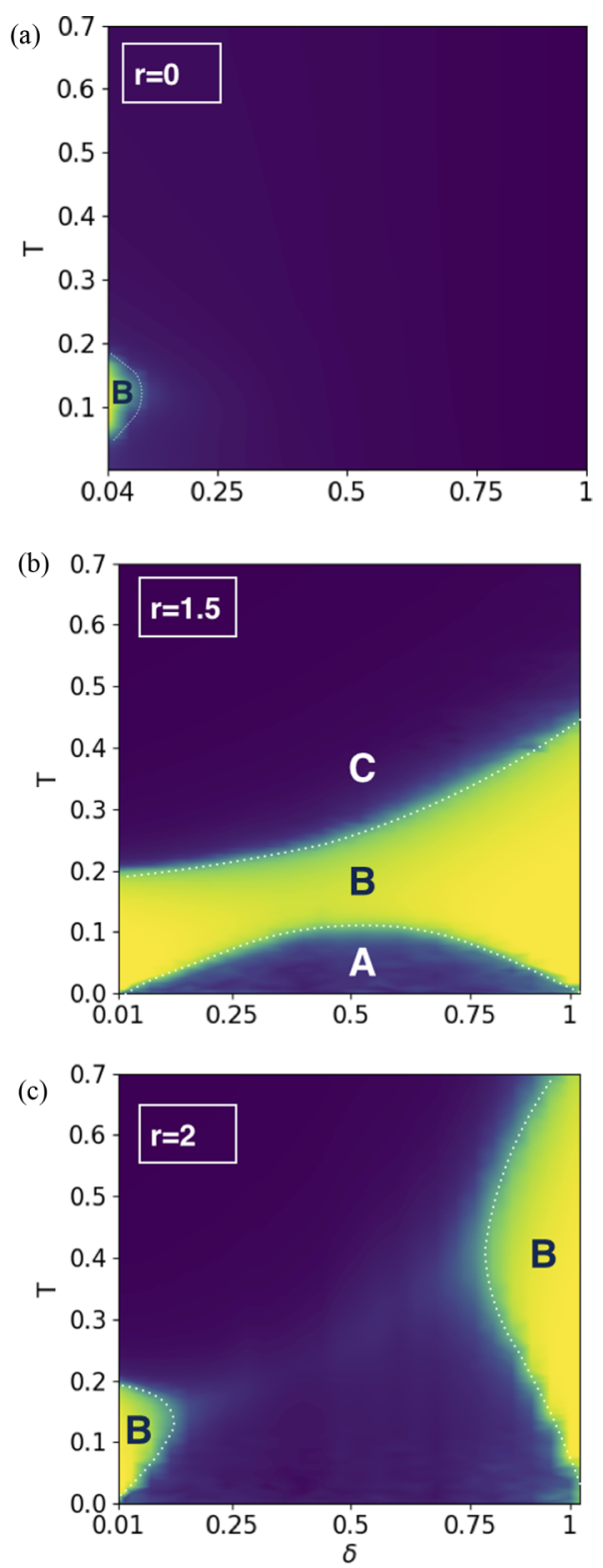

FIG. 1. Color maps of the Ising $\left(M_{Z_{2}}\right)$ order parameter as a function of temperature and dilution for a $L \times L=50 \times 50$ lattice. The system is doped with vacancies $(r=0)$ (a) and with magnetic impurities with $r=1.5$ (b) and $r=2$ (c) being $r=S_{\text {imp }} / S_{\text {lat }}$. Colors range from blue (minimum) to yellow (maximum). From low to high temperature different ordered regions can be distinguished: (A) anticollinear, (B) collinear, and (C) paramagnetic. The white dotted line is a guide to the eyes. $\left(J_{2} / J_{1}=0.55\right.$. $)$

This is expected for the former case (nonmagnetic dopants $r=0$ ), where large dilutions prevents the propagation of long-range magnetic order as the magnetic order propagates by short-range correlations. The quenching of low-energy fluctuations upon the introduction of nonmagnetic impurities have been observed experimentally both in vanadates [39] and pnictides [11].

For the latter case (magnetic dopants with $r=2$ ) the drop of the Ising order, for fixed temperature and increased dilution, is driven by a different mechanism. Indeed as the collinear
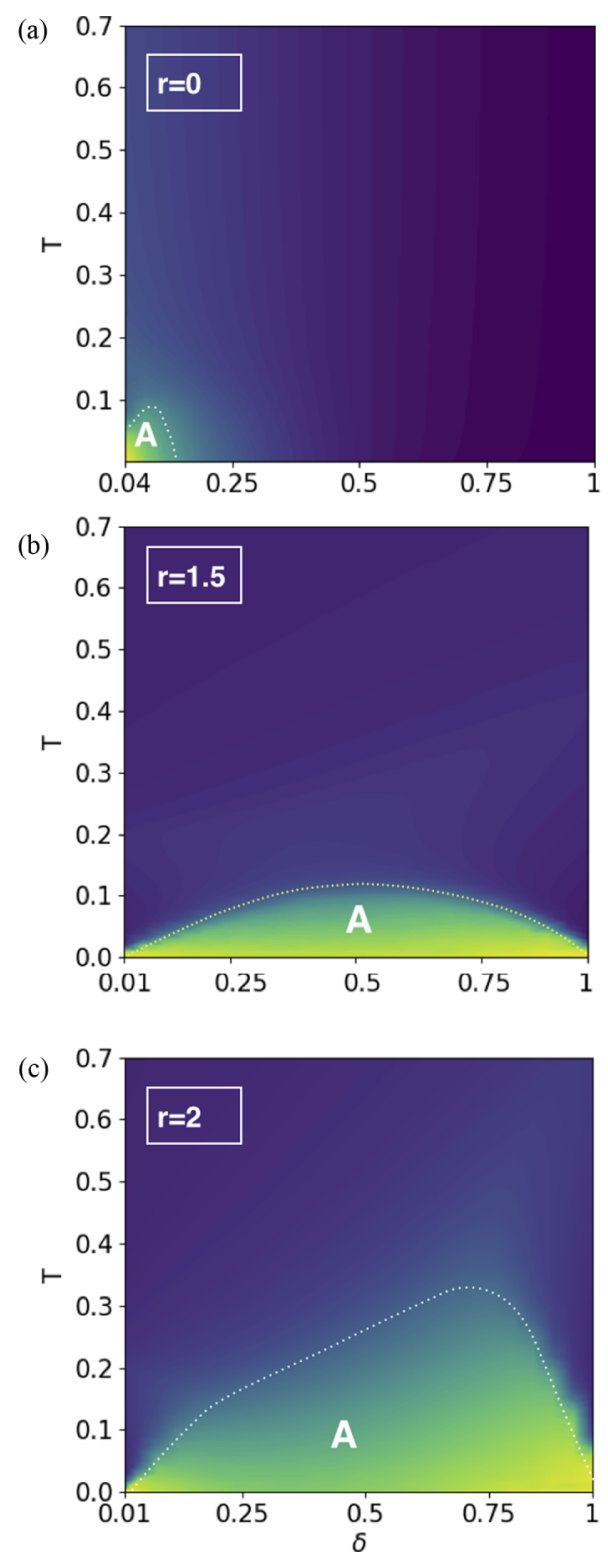

FIG. 2. Color maps of the anticollinear $\left(M_{90}\right)$ order parameter as a function of temperature and dilution for a $L \times L=50 \times 50$ lattice. The system is doped with vacancies $(r=0)$ (a) and with magnetic impurities with $r=1.5$ (b) and $r=2$ (c) being $r=S_{\text {imp }} / S_{\text {lat }}$. Colors range from blue (minimum) to yellow (maximum). From low to high temperature different ordered regions can be distinguished: (A) anticollinear, (B) collinear, and (C) paramagnetic. The white dotted line is a guide to the eyes. $\left(J_{2} / J_{1}=0.55\right.$. $)$

order disappears a new competing order appears [Fig. 2(b)]. The same observation is valid for $r=1.5$ [Fig. 1(b)] at low temperatures. Indeed if we look at fixed dilution, $\delta=50 \%$, we observe that the collinear order is also suppressed at $T<0.1\left[J_{1}\right]$ (region A), and we obtain a reentrance transition of the collinear order (region A to B). This is expected at low temperature and low doping; indeed it has been shown that around a single impurity the degeneracy of the ground state of the $J_{1}-J_{2}$ model is lifted and the $90^{\circ}$ magnetic order is selected from the manifold by an energy optimization process $[40,41]$. Note that this latter mechanism is driven by 


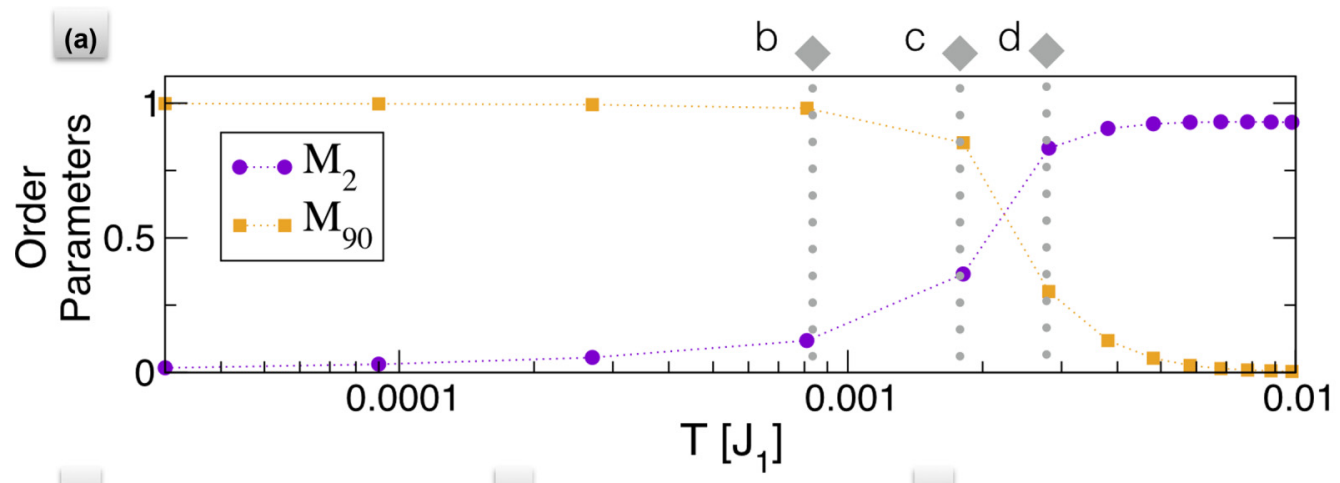

(b)
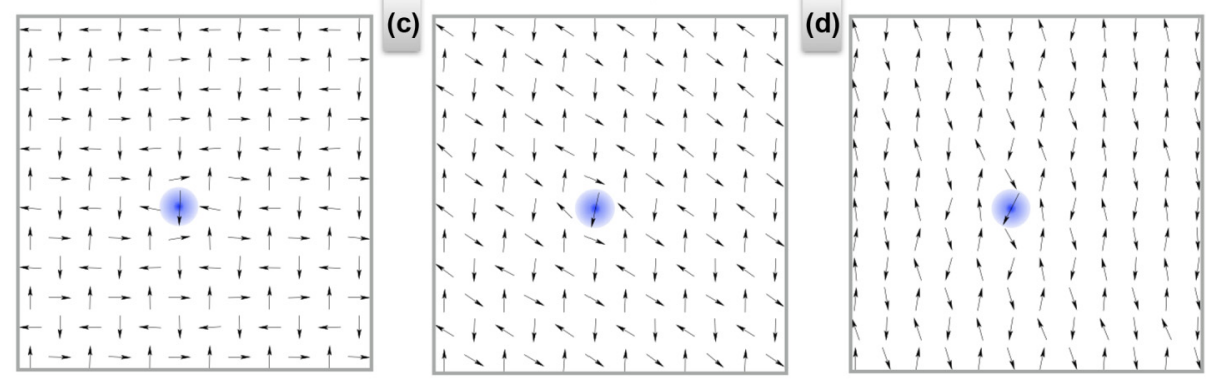

- $-\mathrm{T}=0.0001$

$\bullet \cdot \mathrm{T}=0.001$

$\Delta \cdots . \mathrm{T}=0.01$
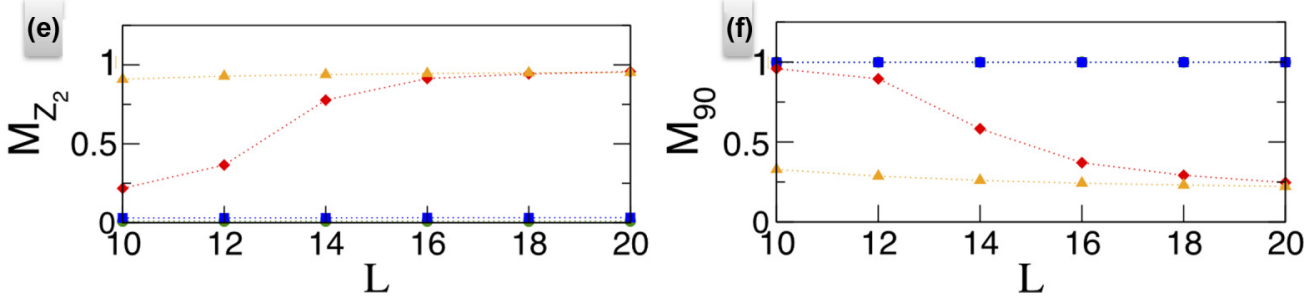

FIG. 3. Case of doping with single magnetic impurity with ratio $r=S_{\text {imp }} / S_{\text {lat }}=1.5$. (a) Temperature dependence of $M_{2}$ and $M_{90}$ for a lattice with $L=12$. (b)-(d) Typical spin configuration obtained at three fixed temperatures, of panel (a), at $T=0.0008,0.00181,0.00281 J_{1}$. (e) and (f) are respectively the collinear $M_{Z_{2}}$ and anticollinear $M_{90^{\circ}}$ order parameter values for fixed temperatures $T=0.0001,0.001,0.01 J_{1}$ as a function of different lattices with linear dimension $L$. We fixed $J_{2} / J_{1}=0.55$ and $\delta=1 / L^{2}$.

an energy optimization and is not expected to survive to high temperatures.

Effect of doping on the anticollinear order. In Figs. 2(a) and 2(b) we report the anticollinear order parameter

$$
M_{90}(x)=\left|\left(\hat{\mathbf{S}}_{i}-\hat{\mathbf{S}}_{k}\right) \times\left(\hat{\mathbf{S}}_{j}-\hat{\mathbf{S}}_{l}\right)\right|,
$$

where $(i, j, k, l)$ defines the same plaquette as in Eq. (2) [see Fig. S1(a)]. Our results confirm that the order stabilized in region $A$ in Fig. 2(b) is the $90^{\circ}$ order. Local fluctuations of the $90^{\circ}$ order around impurities percolate and form a stable order at low temperature. At high temperature the entropic contribution dominates and the Ising-like order is recovered [Fig. 1(b)]. Note, however, that if the magnetic moment of the dopant is large $(r=2)$, the entropic contributions are not able to recover the collinear order and the $90^{\circ}$ order surprisingly stabilizes at high temperature until the paramagnetic phase is obtained [Fig. 2(c)], leading to a suppression of the Ising order in between the undoped and fully doped regions. This has been observed in the superconducting pnictides doped with Ir [15] where the collinear order is suppressed when the dilution is greater than $\delta>0.047$. This is in agreement with the quenching of the collinear phase observed with impurity ratio $r=2$ in the doping region $\delta=[0.2,0.8]$. Indeed in
Fig. 2(c) we can clearly see that at approximatively half doping the low-temperature range is fully dominated by the anticollinear order $\left(M_{90}\right)$ being the collinear order, $\left(M_{Z_{2}}\right)$ equal to zero [Fig. 1(c)]. Note that this mechanism is not obtained by doping with nonmagnetic impurities [Figs. 1(a) and 2(a)], as the suppression of the Ising-like order is not concomitant with the stabilization of a competing order.

In the dilution range $\delta<0.2$ and $\delta>0.8$ (case $r=2$ ), the competition between the entropic and the energetic contribution is restored and interestingly we observed that the reentrance transition [region A to B, Figs. 1(b) and 2(a), low dilution] is characterized by a sharp crossover.

\section{ANALYSIS OF THE REENTRANCE PHASE TRANSITION}

The reentrance phenomena and the transition between the collinear $M_{Z_{2}}$ and anticollinear $M_{90}$ order can be explained on the basis of the single-impurity results at finite temperature. We observe in Fig. 3(a) that there is a sharp crossover between $M_{90}$ and $M_{Z_{2}}$. This suggests that there is a crossing of the free energies of the $90^{\circ}$ and collinear orders at the transition, where the competition in the free energies $F=E-T * S$ happens 

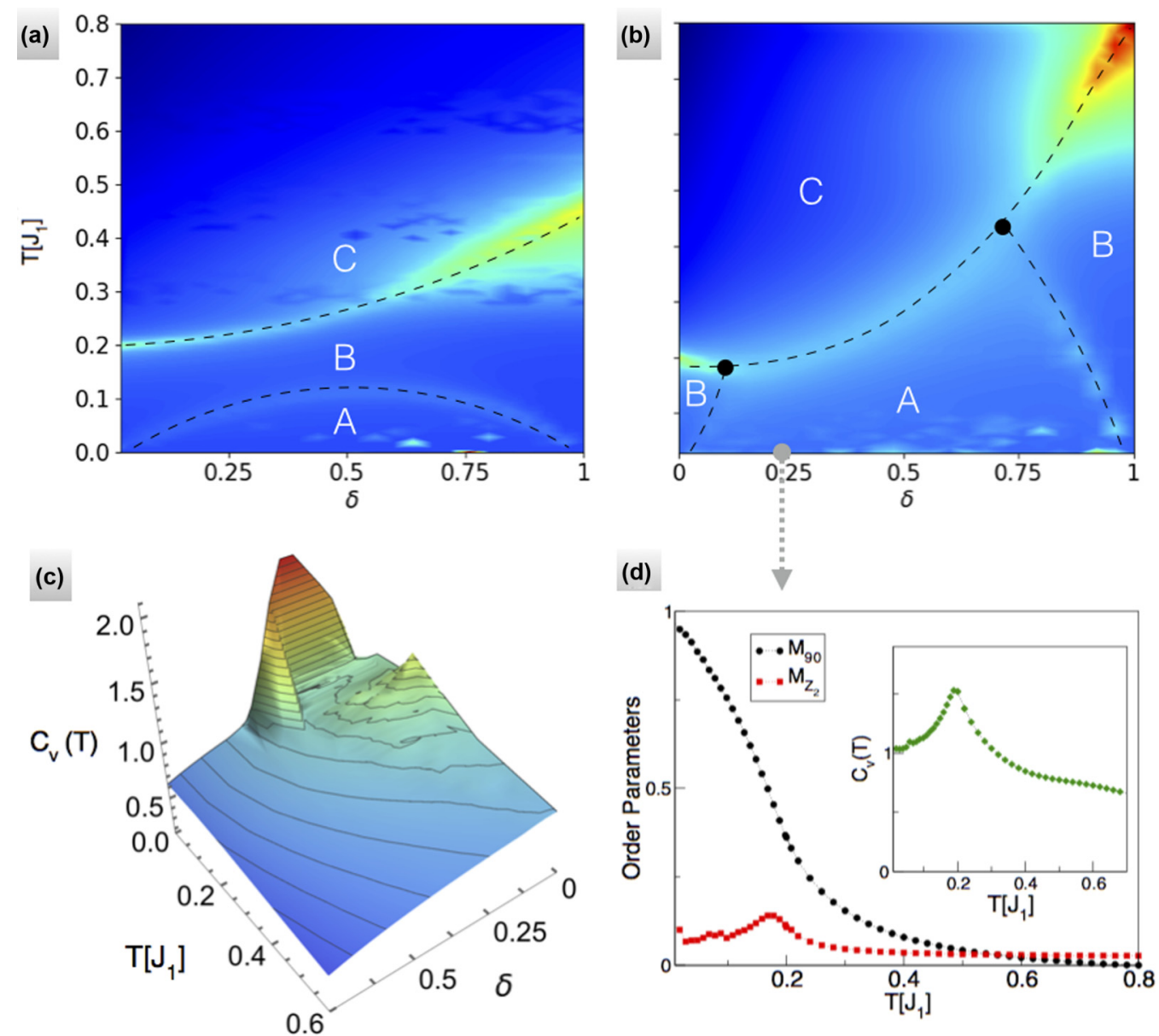

FIG. 4. Color maps of the specific heat as a function of temperature and dilution for a $L \times L=50 \times 50$ lattice respectively for magnetic impurities $r=1.5$ (a), $r=2$ (b), and $r=0$ (c). Colors range from blue (minimum) to red (maximum). Black dashed lines are a guide to the eyes to distinguish the three ordered states: A (anticollinear), B (collinear), and C (paramagnetic). (d) shows the temperature-dependent behavior of the anticollinear (black dotted line), the collinear (red dotted line) order parameter, and specific heat (green dotted line in the inset) for fixed dilution $\delta=25 \%$ and $r=2$. $\left(J_{2} / J_{1}=0.55\right.$. $)$

between the energy term $E$ and entropic contribution $T * S$. As this process is very much dependent on the local disorder configurations, the temperature associated with the sharp crossover is also dependent on the disorder configurations. In an experiment, or in our computed physical observables which are averaged over large disorder samples, the transition is a smooth crossover, hiding the physical explanation related to the competition of energetic and entropic terms.

Remarkably, the mechanism which determines the energy vs entropy competition is different respectively to vacancies or magnetic doping. Indeed in the case of nonmagnetic impurity the transition between the anticollinear and the collinear phase is happening through a coexistent phase: it was shown (Ref. [26]) that at finite temperature the anticollinear order stabilizes locally around the impurity and with the collinear states recovered outside this region. Instead, in the case of magnetic impurities, we observe that the magnetic phase which characterizes the crossover is not a coexistent phase of collinear and anticollinear order. Indeed looking at Fig. 3(c) we observe that there exists a magnetic phase different from both the anticollinear [Fig. 3(b)] and the collinear case [Fig. 3(d)]. This intermediate phase consists of two distinct antiferromagnetically ordered states on two sublattices with a relative angle $\alpha$ between their magnetization axis, which is selected by the impurity spin direction.

The transition between the $90^{\circ}$ and collinear order is rationalized with respect to the lattice size in Figs. 3(e) and 3(f), where we show the order parameters at three different temperatures, $T=10^{-4}, 10^{-3}, 10^{-2} J_{1}$, for a single impurity embedded in a lattice of size from $L=10$ to 20 . Note that periodic conditions are used in this simple model, such that the lattice size mimics the average distance between impurities at high dilutions. At low temperature $T=10^{-4}$, as entropic contributions are absent, we observe that the $90^{\circ}$ order dominates as expected for all cases [analytic argument at $T=0$ in Supplemental Material Fig. S1(b)]. As temperature is increased to $T=10^{-3}$ and $T=10^{-2}$, we observe that the $90^{\circ}$ is stabilized at small $L$, but the collinear order wins in larger lattices where the entropic contributions in turn become larger. This illustrates the mechanism obtained around the large dilution (small $L$ ), where the $90^{\circ}$ order is stabilized, and at low dilutions ( $\operatorname{large} L$ ), where the collinear order wins.

Further insights about the transition between the different magnetic phases are shown in Fig. 4. For doping with magnetic impurities [Fig. 4(a)], we obtain as expected a large peak in the specific heat at the transition associated with 
the loss of the collinear order [region B to C, Fig. 1(b)]. As we do not observe a drop in the specific heat along the Ising-like transition in Fig. 4(a) (where $r=1.5$ ), we conclude that the transition remains second order along this line. Surprisingly a continuous transition occurs also in Fig. 4(b) (with $r=2$ ) between the anticollinear and the paramagnetic phase, even if the Ising-like order is zero for all $T$. In more detail we observe in Fig. 4(d) that at fixed dilution $\delta=25 \%$ the melting of the anticollinear order occurs with a continuous crossover associated with a nondivergent peak of the specific heat (further details are shown in the Supplemental Material Figs. S4 and S5). Note that the specific heat also indicates weak fluctuations at the reentrance transition (region A to B) which are, however, of a different nature since related to the sharp crossover between the collinear and the anticollinear phase (see Supplemental Material Fig. S5.)Thus for $r=2$ we individuate the crossing of the three different magnetic phases [anticollinear (A), collinear (B), and paramagnetic (C)].

For doping with nonmagnetic impurities Fig. 4(c) we observe the irising of the peaks for the Ising-like transition (fixed low doping), which is consistent with what is observed so far in the case of magnetic impurities. A more interesting and novel behavior is observed at fixed low temperature where there exists a continuous pathway which does not involve any sharp transition. This is crucial for applications because it does not involve any energy cost. This was not observed in the previous work because no fluctuations were considered. At zero temperature, we observe that there are no energy fluctuations associated with the percolation transition which is instead indicated by the sudden disappearance of the susceptibility at $8 \%$ in Supplemental Material Fig. S6, typical of a first-order transition.

\section{CONCLUSION}

In conclusion we found that the order-by-disorder entropy selection, associated with the Ising-like phase transition that appears for $J_{2} / J_{1}>1 / 2$ in the pure spin model, is quenched at low temperature due to the presence of impurities. Indeed, irrespective of the magnetic ratio of the dopant an anticollinear order is stabilized around the impurities, which in turn induces a reentrance of the Ising-like phase transition. The melting of the collinear order occurs via two different mechanisms: (i) through a percolation transition from increasing dilution (at fixed temperature) and (ii) via a sharp crossover due to the energetic versus entropic contribution increasing temperature (at fixed doping). While the former exists irrespective to the nature of the dopant the latter is highly affected by the ratio of the magnetic impurities. Remarkably we identify a regime where the anticollinear order is stabilized at finite temperature without going through the collinear phase.

\section{ACKNOWLEDGMENTS}

We thank R. Fernandes and P. Carretta for insighful suggestions following their critical reading of the manuscript. C.L. is supported by the EPSRC Centre for Doctoral Training in Cross-Disciplinary Approaches to Non-Equilibrium Systems (CANES, EP/L015854/1). C.W. gratefully acknowledges the support of NVIDIA Corporation, ARCHER UK National Supercomputing Service. We are grateful to the Hartee Centre (Bluegene -Q) for computational resources. This work was supported by EPSRC (EP/R02992X/1).
[1] Y. Takabayashi, A. Y. Ganin, P. Jeglic, D. Arcon, T. Takano, Y. Iwasa, Y. Ohishi, M. Takata, N. Takeshita, K. Prassides, and M. J. Rosseinsky, Science 323, 1585 (2009).

[2] T. Park, F. Ronning, H. Q. Yuan, M. B. Salamon, R. Movshovich, J. L. Sarrao, and J. D. Thompson, Nature (London) 440, 65 (2006).

[3] Y. Kamihara, T. Watanabe, M. Hirano, and H. Hosono, J. Am. Chem. Soc. 130, 3296 (2008).

[4] J.-H. Chu, J. G. Analytis, K. De Greve, P. L. McMahon, Z. Islam, Y. Yamamoto, and I. R. Fisher, Science 329, 824 (2010).

[5] T.-M. Chuang, M. P. Allan, J. Lee, Y. Xie, N. Ni, S. L. Bud'ko, G. S. Boebinger, P. C. Canfield, and J. C. Davis, Science 327, 181 (2010).

[6] P. Richard, K. Nakayama, T. Sato, M. Neupane, Y.-M. Xu, J. H. Bowen, G. F. Chen, J. L. Luo, N. L. Wang, X. Dai, Z. Fang, H. Ding, and T. Takahashi, Phys. Rev. Lett. 104, 137001 (2010).

[7] W. Lv, F. Krüger, and P. Phillips, Phys. Rev. B 82, 045125 (2010).

[8] B. Valenzuela, E. Bascones, and M. J. Calderón, Phys. Rev. Lett. 105, 207202 (2010).

[9] D. K. Pratt, M. G. Kim, A. Kreyssig, Y. B. Lee, G. S. Tucker, A. Thaler, W. Tian, J. L. Zarestky, S. L. Bud'ko, P. C. Canfield, B. N. Harmon, A. I. Goldman, and R. J. McQueeney, Phys. Rev. Lett. 106, 257001 (2011).
[10] S. Mukhopadhyay, S. Oh, A. M. Mounce, M. Lee, W. P. Halperin, N. Ni, S. L. Bud'ko, P. C. Canfield, A. P. Reyes, and P. L. Kuhns, New J. Phys. 11, 055002 (2009).

[11] P. Bonfà, P. Carretta, S. Sanna, G. Lamura, G. Prando, A. Martinelli, A. Palenzona, M. Tropeano, M. Putti, and R. De Renzi, Phys. Rev. B 85, 054518 (2012).

[12] C.-C. Chen, R. Applegate, B. Moritz, T. P. Devereaux, and R. R. P. Singh, New J. Phys. 13, 043025 (2011).

[13] M. Moroni, P. Carretta, G. Allodi, R. De Renzi, M. N. Gastiasoro, B. M. Andersen, P. Materne, H.-H. Klauss, Y. Kobayashi, M. Sato, and S. Sanna, Phys. Rev. B 95, 180501(R) (2017).

[14] M. N. Gastiasoro, F. Bernardini, and B. M. Andersen, Phys. Rev. Lett. 117, 257002 (2016).

[15] M. P. M. Dean, M. G. Kim, A. Kreyssig, J. W. Kim, X. Liu, P. J. Ryan, A. Thaler, S. L. Bud'ko, W. Strassheim, P. C. Canfield, J. P. Hill, and A. I. Goldman, Phys. Rev. B 85, 140514(R) (2012).

[16] W. R. Meier, Q.-P. Ding, A. Kreyssig, S. L. Bud'ko, A. Sapkota, K. Kothapalli, V. Borisov, R. Valentí, C. D. Batista, P. P. Orth, R. M. Fernandes, A. I. Goldman, Y. Furukawa, A. E. Böhmer, and P. C. Canfield, npj Quantum Mater. 3, 5 (2018).

[17] X. Wang, J. Kang, and R. M. Fernandes, Phys. Rev. B 91, 024401 (2015). 
[18] R. M. Fernandes, S. A. Kivelson, and E. Berg, Phys. Rev. B 93, 014511 (2016).

[19] Q. Si and E. Abrahams, Phys. Rev. Lett. 101, 076401 (2008).

[20] P. Chandra and B. Doucot, Phys. Rev. B 38, 9335 (1988).

[21] J. Zhao, D. T. Adroja, D.-X. Yao, R. Bewley, S. Li, X. F. Wang, G. Wu, X. H. Chen, J. Hu, and P. Dai, Nat. Phys. 5, 555 (2009).

[22] A. L. Wysocki, K. D. Belashchenko, and V. P. Antropov, Nat. Phys. 7, 485 (2011).

[23] R. A. Ewings, T. G. Perring, J. Gillett, S. D. Das, S. E. Sebastian, A. E. Taylor, T. Guidi, and A. T. Boothroyd, Phys. Rev. B 83, 214519 (2011).

[24] I. Eremin and A. V. Chubukov, Phys. Rev. B 81, 024511 (2010).

[25] C. Cao and J. Dai, Chin. Phys. Lett. 28, 057402 (2011).

[26] C. Weber and F. Mila, Phys. Rev. B 86, 184432 (2012).

[27] G. Franzese, V. Cataudella, S. E. Korshunov, and R. Fazio, Phys. Rev. B 62, 9287(R) (2000).

[28] C. Weber, L. Capriotti, G. Misguich, F. Becca, M. Elhajal, and F. Mila, Phys. Rev. Lett. 91, 177202 (2003).

[29] C. Weber, F. Becca, and F. Mila, Phys. Rev. B 72, 024449 (2005).
[30] See Supplemental Material at http://link.aps.org/supplemental/ 10.1103/PhysRevB.99.214439 for the description of the numerical methods implemented.

[31] R. H. Swendsen and J.-S. Wang, Phys. Rev. Lett. 57, 2607 (1986).

[32] M. Doxastakis, Y.-L. Chen, O. Guzmán, and J. J. de Pablo, J. Chem. Phys. 120, 9335 (2004).

[33] W. Im and C. L. Brooks, J. Mol. Biol. 337, 513 (2004).

[34] H. G. Katzgraber, M. Palassini, and A. P. Young, Phys. Rev. B 63, 184422 (2001).

[35] Y. Xu and D.-X. Yao, Phys. Rev. B 97, 224419 (2018).

[36] J. A. Olive, A. P. Young, and D. Sherrington, Phys. Rev. B 34, 6341 (1986).

[37] Y. Miyatake, M. Yamamoto, J. J. Kim, M. Toyonaga, and O. Nagai, J. Phys. C 19, 2539 (1986).

[38] P. Chandra, P. Coleman, and A. I. Larkin, Phys. Rev. Lett. 64, 88 (1990).

[39] R. Melzi, P. Carretta, A. Lascialfari, M. Mambrini, M. Troyer, P. Millet, and F. Mila, Phys. Rev. Lett. 85, 1318 (2000).

[40] C. Henley, J. Appl. Phys. 61, 3962 (1987).

[41] C. L. Henley, Phys. Rev. Lett. 62, 2056 (1989). 